\title{
Combination of the top-quark mass measurements from the Tevatron and from the LHC colliders
}

\section{Frédéric DELIOT*}

On behalf of the ATLAS, CDF, CMS and D0 Collaborations

CEA-Saclay, Irfu/SPP, France

E-mail: frederic.deliot@cea.fr

I present here the results for the combination of the top-quark mass $\left(m_{t}\right)$ measurements at the Tevatron and at the LHC. These combinations are based on measurements using up to $5.8 \mathrm{fb}^{-1}$ from CDF and D0 yielding $m_{t}=173.18 \pm 0.56$ (stat) \pm 0.75 (syst) $\mathrm{GeV}$, and up to $4.7 \mathrm{fb}^{-1}$ from ATLAS and CMS leading to $m_{t}=173.3 \pm 0.5$ (stat) \pm 1.3 (syst) GeV.

36th International Conference on High Energy Physics,

July 4-11, 2012

Melbourne, Australia

${ }^{*}$ Speaker. 


\section{Introduction}

The top quark is a unique particle. Indeed it is the heaviest known elementary particle and it has a Yukawa coupling to the Higgs boson close to unity, which may indicate that it plays a special role in the electroweak symmetry breaking mechanism. It is the only quark that decays before hadronizing, which gives the unique opportunity to study a bare quark. At hadron colliders, it is mainly produced in pairs by the quantum chromodynamic interaction $(\mathrm{QCD})$ via quark-antiquark annihilation ( $q \bar{q} \rightarrow t \bar{t}$, dominant at the Tevatron) or gluon fusion ( $g g \rightarrow t \bar{t}$, dominant at the LHC). Within the Standard Model (SM), the top quark decays almost $100 \%$ of the time into a $W$ boson and a $b$ quark. The $t \bar{t}$ signatures are therefore classified according to the decays of the $W$ bosons into the lepton+jets channel $(\ell+$ jets $)$, the dilepton channel $(\ell \ell)$, the alljets channel and the $B_{T}+$ jets channel.

\section{The top-quark mass}

The top-quark mass is a free parameter of the SM. It should be measured experimentally with a high precision because it enters into the computation of quantum loop corrections for several observables. In particular the $W$ boson mass $\left(M_{W}\right)$ receives radiative corrections proportional to $m_{t}^{2}$ and to the logarithm of the Higgs boson mass $\left(m_{H}\right)$. Therefore measuring $M_{W}$ and $m_{t}$ allows to indirectly constrain $m_{H}$ and consequently to test the consistency of the SM by comparing direct and indirect determination of $m_{H}$. The latest indirect constraint on $m_{H}$ through a fit of the electroweak precision data yields: $m_{H}=94_{-24}^{+29} \mathrm{GeV}$ or $m_{H}<152 \mathrm{GeV}$ at $95 \%$ confidence level [1].

There are three main methods to measure $m_{t}$ directly. The simplest method is called the template method. It relies on a given observable in data sensitive to $m_{t}$ (which is often the reconstructed $m_{t}$ from the top-quark decay products) which is compared to distributions (templates) produced with Monte Carlo (MC) simulation generated with different $m_{t}$ input values. The matrix element method is based on the construction of a per-event probability computed with the leading-order $t \bar{t}$ matrix element using the full event kinematic informations. Finally the ideogram method uses an event likelihood computed as a Gaussian resolution function with a Breit-Wigner to model the top-quark signal. For channels that contain at least one $W$ boson that decays hadronically, the jet energy scale (JES) can be calibrated by constraining the invariant mass of the two light jets to the world-average value of $M_{W}$. This allows to limit the impact of the JES uncertainty on the measured $m_{t}$. In order to correct for approximations and for any potential biases, all methods are calibrated using MC samples.

\section{Top-quark combination at the Tevatron}

The Tevatron experiments have published several measurements of $m_{t}$ over the last twenty years using Run I (1992-1996) and Run II (2001-2011) data sets in 6 top-quark decay channels and different measurement techniques. Twelve measurements that use up to $5.8 \mathrm{fb}^{-1}$ of data are used for the combination. The best independent measurements per channel in each experiment are chosen, eight from CDF and four from D0. The inputs are $5 \ell+$ jets measurements (CDF and D0, Run II and Run I, and a CDF Run II result based on the decay length of B hadrons); 2 alljets 


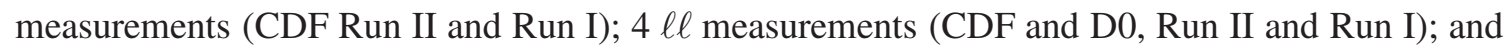
a $\not_{T}+$ jets measurement (CDF Run II).

The combination uses the Best Unbiased Linear Estimate (BLUE) [2,3] method that calculates the combined mass value $m_{t}^{\text {comb }}$ (an estimator of the true top quark mass) as a linear weighted sum of the input results $m_{t}^{i}: m_{t}^{\mathrm{comb}}=\sum_{i=1}^{12} w_{i} m_{t}^{i}$, where the weights are computed as:

$$
w_{i}=\frac{\sum_{j=1}^{12} \text { Covariance }^{-1}\left(m_{t}^{i}, m_{t}^{j}\right)}{\sum_{i=1}^{12} \sum_{j=1}^{12} \text { Covariance }^{-1}\left(m_{t}^{i}, m_{t}^{j}\right)},
$$

and Covariance ${ }^{-1}\left(m_{t}^{i}, m_{t}^{j}\right)$ are the elements of the inverse of the covariance matrix (i.e. the error matrix) of the input measurements. The uncertainties on the 12 inputs are separated into 15 parts to properly estimate this covariance matrix getting the correct pattern of correlation between channels, run periods and experiments. After several years of discussion, the CDF and D0 collaborations have agreed on a common list of systematics, on common evaluations and splitting of the systematic uncertainties and on their correlations. This common splitting is described in the following.

The systematic uncertainties from JES are the largest sources of systematic uncertainties on the measured $m_{t}$ inputs. These are split into 7 different parts:

- Uncertainty from light-jet response (1) (also called rJES): this part is specific to CDF measurements and comes from CDF method of calibrating JES using single-pion response in data and in MC. It is implemented by tuning the simulation and is assumed to be $100 \%$ correlated between all CDF measurements;

- Uncertainty from light-jet response (2) (also called dJES): this part corresponds to the absolute and relative uncertainty on JES calibration using $\gamma+$ jets events in D0 and $\eta$-dependent calibration in CDF. It is $100 \%$ correlated within the same experiment and the same run period;

- Uncertainty from out-of-cone corrections (also called cJES): this is the uncertainty coming from out-of-cone corrections to MC showers for CDF and D0 Run I measurements. It is $100 \%$ correlated between all measurements;

- Uncertainty from offset (also called UN/MI): this part arises from the uncertainty on JES coming from the uranium decay noise and pile-up from previous collisions. Due to the smaller integration time for D0 calorimeter electronics in Run II, it is only a significant source of uncertainty in D0 Run I measurements. It is $100 \%$ correlated within D0 Run I measurements;

- Uncertainty from modeling of $b$-jets (also called bJES): this part corresponds to the difference between models of $b$-jet hadronization. It is $100 \%$ correlated between all measurements;

- Uncertainty from the response difference for $b$-, $q$ - and $g$-jets (also called aJES): it arises from $\mathrm{MC} /$ data difference in response between $b$-jets, light and gluon jets. It is assumed to be $100 \%$ correlated within the same experiment and the same run period; 
- Uncertainty from in-situ light-jet calibration (also called iJES): this part is relevant for channels with at least one $W$ boson decaying hadronically that uses the light dijet invariant mass to calibrate the JES. It is $100 \%$ uncorrelated between all measurements and is scaling with the statistical uncertainty of the amount of analyzed data.

The other sources of systematic uncertainties that are not related with JES are also split into 7 parts:

- Uncertainty from jet modeling: this part arises from the uncertainty on jet identification efficiency and jet smearing at D0. It is $100 \%$ correlated between all D0 Run II measurements;

- Uncertainty from lepton modeling: it comes from the uncertainty on electron and muon momentum scale, including also the uncertainty on the muon momentum smearing at D0. It is assumed to be $100 \%$ correlated within the same experiment and the same run period;

- Uncertainty from signal modeling: this part contains the uncertainty coming from the limited knowledge on the parton distribution functions and on the $q \bar{q} / g g$ fraction in the $t \bar{t}$ production, from corrections due to higher-order QCD, from uncertainties on the initial and final-state radiation modeling, from the hadronization model and from color reconnection. It is $100 \%$ correlated between all measurements;

- Uncertainty from the multiple interaction model: it arises from the uncertainty on the modeling of pile-up in the MC and is $100 \%$ correlated within the same experiment and the same run period;

- Uncertainty from the background coming from theory: this part contains the uncertainty on the NLO fraction of heavy flavor jets in the $W+$ jets MC samples, the uncertainty from factorization and renormalization scales in the $W+$ jets simulation and from theory cross sections used to normalize the MC samples. It is assumed to be $100 \%$ correlated between all measurements in the same channel;

- Uncertainty from background estimation based on data: this uncertainty comes from the $\mathrm{MC} /$ data difference in some background distributions and from the signal/background fraction. It is $100 \%$ correlated within the same experiment and the same run period in the same channel;

- Uncertainty from the calibration method: it arises from the uncertainty on the calibration curve and is uncorrelated between all measurements.

With this splitting of the systematic uncertainties and the above correlations, the combined value for the Tevatron top-quark mass is: $m_{t}=173.18 \pm 0.56$ (stat) \pm 0.75 (syst) GeV corresponding to a total uncertainty of $0.94 \mathrm{GeV}$ and a total relative error of $0.54 \%$ [4]. The $\chi^{2}$ for this combination is 8.3 for 11 degrees of freedom which is equivalent to a $69 \%$ probability for agreement among the 12 input measurements. Figure 1 shows this combined value together with the input measurements. In the combination, the input measurements that get the largest weights are CDF and D0 Run II $\ell+$ jets measurements (55.5\% and 26.7\%) and CDF Run II measurement in the alljets channel (14\%). The main uncertainties in the combined value are the statistical uncertainty 
$(0.56 \mathrm{GeV})$, the systematic uncertainty from in-situ light-jet calibration $(0.39 \mathrm{GeV})$ and from signal modeling $(0.51 \mathrm{GeV})$. The two first ones will scale down when all the Tevatron data will be analyzed. We can expect therefore that the final combination of the top-quark mass measurement at the Tevatron will have a total uncertainty around $0.7-0.8 \mathrm{GeV}$.

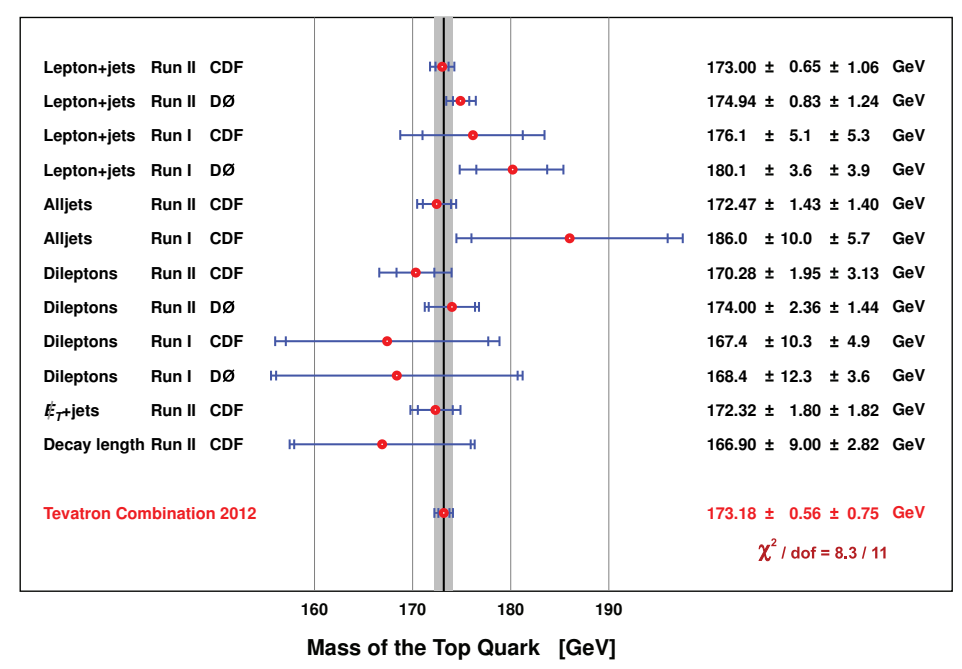

Figure 1: The 12 input measurements of $m_{t}$ from the Tevatron collider experiments along with the resulting combined value [4].

\section{Top-quark combination at the LHC}

At the LHC, 7 input measurements are used in the combination. All were obtained by analyzing LHC data at $7 \mathrm{TeV}$. These inputs are ATLAS [5] 2010 measurement in the $\ell+$ jets channel using $35 \mathrm{pb}^{-1}$, ATLAS $2011 \ell$ +jets measurement using $1.0 \mathrm{fb}^{-1}$, ATLAS 2011 measurement in the alljets channel using $2.0 \mathrm{fb}^{-1}$, CMS [6] 2010 measurement in the $\ell \ell$ channel using $36 \mathrm{pb}^{-1}$, CMS $2010 \ell+$ jets measurement using $36 p b^{-1}$, CMS $2011 \ell \ell$ measurement using $2.3 \mathrm{fb}^{-1}$ and CMS 2011 measurement in the $\mu+$ jets channel using $4.7 \mathrm{fb}^{-1}$.

The systematic uncertainties on these measurements are split using the same categories as described for the Tevatron in the previous section. However two categories are not used (aJES and cJES, the latter being included into dJES) but two additional categories are taken into account. Since the uncertainties from initial- and final-state radiations and from hadronization are assumed to be $50 \%$ correlated between the LHC experiments, these are separated from the signal modeling systematic uncertainty which is taken to be $100 \%$ correlated. A new category also includes the uncertainty from the modeling of underlying event, which was not considered as a separate source at the Tevatron but included in the uncertainty from signal modeling.

The LHC top-quark mass combination result yields: $m_{t}=173.3 \pm 0.5$ (stat) \pm 1.3 (syst) GeV corresponding to a total uncertainty of $1.4 \mathrm{GeV}$ [7]. The $\chi^{2}$ for this combination is 2.5 for 6 degrees of freedom which is equivalent to a $86 \%$ probability for agreement among the input measurements. Figure 2 shows this combined value together with the input measurements at the LHC. The largest weights in the combination are carried by the CMS $2011 \mu+$ jets measurement $(65.7 \%)$ and the 
ATLAS $2011 \ell+$ jets measurement (23.3\%). The assumed correlations between the systematic uncertainties were checked by varying the fully correlated sources from the default value of $100 \%$ to $0 \%$ simultaneously in steps of $10 \%$. This lead to a variation of the combination central value of less than $200 \mathrm{MeV}$. The largest uncertainties in the combination arise from the uncertainty on initial- and final-state radiation $(0.69 \mathrm{GeV})$ and from color reconnection $(0.55 \mathrm{GeV})$ as well as from uncertainty in the modeling and response from $b$-jets (bJES: $0.68 \mathrm{GeV}$ ).

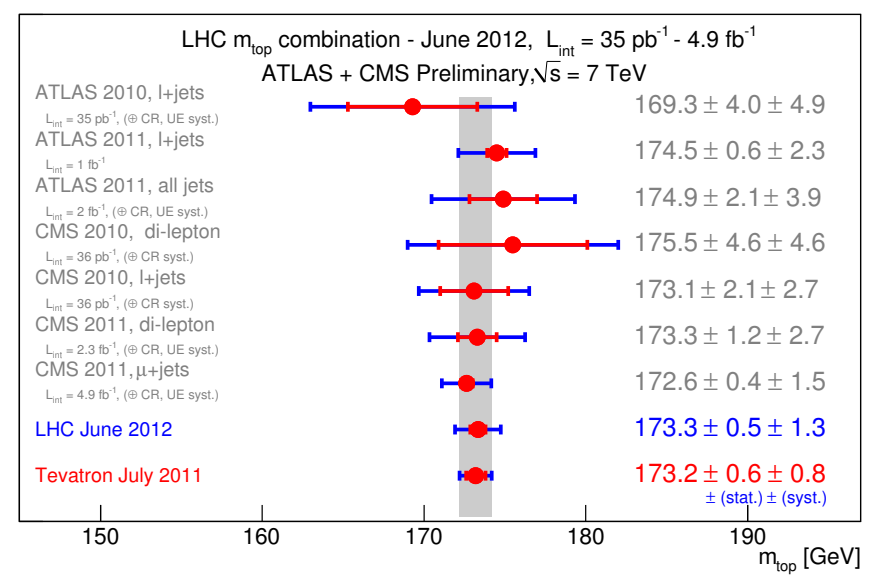

Figure 2: Input measurements and result of the LHC combination [7].

\section{Conclusion}

The combination of the top-quark mass measurements from the Tevatron and from the LHC were presented. The Tevatron combination, which was first published in 2012, has now a total uncertainty below $1 \mathrm{GeV}$ while the uncertainty on the final combination is expected to be around 0.7 to $0.8 \mathrm{GeV}$. The first preliminary combination of the LHC measurements has a total uncertainty of $1.4 \mathrm{GeV}$. With the large amount of $t \bar{t}$ statistics available at the LHC, this uncertainty is expected to decrease by constraining systematic uncertainties directly using data and by performing topquark mass measurements in specific regions of the phase space.

\section{References}

[1] http://lepewwg.web.cern.ch/LEPEWWG/

[2] L. Lyons, D. Gibaut, and P. Clifford, Nucl. Instrum. Methods in Phys. Res. Sect. A 270, 110 (1988).

[3] A. Valassi, Nucl. Instrum. Methods in Phys. Res. Sect. A 500, 391 (2003).

[4] T. Aaltonen et al. [CDF and D0 Collaborations], Phys. Rev. D 86, 092003 (2012).

[5] ATLAS Collaboration, JINST 3, S08003 (2008).

[6] CMS Collaboration, JINST 3, S08004 (2008).

[7] ATLAS and CMS Collaborations, ATLAS-CONF-2012-095, CMS PAS TOP-12-001 (2012), http://cds.cern.ch/record/1460441/ 